\title{
ITERATIVELY SOLVING A KIND OF SIGNORINI TRANSMISSION PROBLEM IN A UNBOUNDED DOMAIN
}

\author{
QIYA $\mathrm{HU}^{1}$ AND DeHAO YU ${ }^{1}$
}

\begin{abstract}
In this paper, we are concerned with a kind of Signorini transmission problem in a unbounded domain. A variational inequality is derived when discretizing this problem by coupled FEM-BEM. To solve such variational inequality, an iterative method, which can be viewed as a variant of the D-N alternative method, will be introduced. In the iterative method, the finite element part and the boundary element part can be solved independently. It will be shown that the convergence speed of this iteration is independent of the mesh size. Besides, a combination between this method and the steepest descent method is also discussed.
\end{abstract}

Mathematics Subject Classification. 65N30, 65R20, 73C50.

Received: April 15, 2004. Revised: April 3, 2005.

\section{Introduction}

In this paper we analyze the following transmission problem in $\mathbf{R}^{2}$. Let $\Omega$ be a bounded domain with Lipschitz boundary $\Gamma$. To describe mixed boundary conditions, let $\Gamma=\overline{\Gamma_{t} \cup \Gamma_{s}}$ where $\Gamma_{t}$ and $\Gamma_{s}$ are nonempty, disjoint, and open in $\Gamma$. Let $n$ denote the unit normal on $\Gamma$ defined almost everywhere pointing from $\Omega$ into $\Omega_{c}:=\mathbf{R}^{2} \backslash \bar{\Omega}$. Assume that $f \in L^{2}(\Omega), u_{0} \in H^{\frac{1}{2}}(\Gamma)$ and $t_{0} \in L^{2}(\Gamma)$.

As the interior part, we consider the nonlinear partial differential equation

$$
\operatorname{div}(p(|\nabla u|) \cdot \nabla u)+f=0 \quad \text { in } \Omega,
$$

where $p:[0, \infty) \rightarrow[0, \infty)$ is a continuous function with $t \cdot p(t)$ being monotonously increasing with $t$. In the exterior part, we consider the Laplace equation

$$
\triangle u=0 \quad \text { in } \Omega_{c}
$$

with the radiation condition (note Lem. 3.6 of [2])

$$
u(x)=a+o(1) \quad(|x| \rightarrow \infty)
$$

Keywords and phrases. Signorini contact, FEM-BEM coupling, variational inequality, D-N alternation, convergence rate.

1 Institute of Computational Mathematics and Scientific/Engineering Computing, Academy of Mathematics, Chinese Academy of Sciences, Beijing 100080, China. hqy@lsec.cc.ac.cn; ydh@lsec.cc.ac.cn

The work of Q. Hu was supported by the National Natural Science Foundation No. 10371129.

The work of D. Yu was supported by the State Major Key Project for Basic Researches of China G19990328.

(C) EDP Sciences, SMAI 2005 
where $a$ is a real constant. Writing $u_{1}:=\left.u\right|_{\Omega}$ and $u_{2}:=\left.u\right|_{\Omega_{c}}$, the traction on $\Gamma$ are given by the traces of $p(|\nabla u|) \frac{\partial u_{1}}{\partial n}$ and $-\frac{\partial u_{2}}{\partial n}$. We consider transmission conditions on $\Gamma_{t}$,

$$
\left.u_{1}\right|_{\Gamma_{t}}=\left.u_{2}\right|_{\Gamma_{t}}+\left.u_{0}\right|_{\Gamma_{t}} \text { and }\left.p(|\nabla u|) \frac{\partial u_{1}}{\partial n}\right|_{\Gamma_{t}}=\left.\frac{\partial u_{2}}{\partial n}\right|_{\Gamma_{t}}+\left.t_{0}\right|_{\Gamma_{t}}
$$

and Signorini conditions on $\Gamma_{s}$,

$$
\begin{gathered}
\left.u_{1}\right|_{\Gamma_{s}} \leq\left. u_{2}\right|_{\Gamma_{s}}+\left.u_{0}\right|_{\Gamma_{s}}, \\
\left.p(|\nabla u|) \frac{\partial u_{1}}{\partial n}\right|_{\Gamma_{s}}=\left.\frac{\partial u_{2}}{\partial n}\right|_{\Gamma_{s}}+\left.t_{0}\right|_{\Gamma_{s}} \leq 0 \\
0=\left.\left.p(|\nabla u|) \frac{\partial u_{1}}{\partial n}\right|_{\Gamma_{s}} \cdot\left(u_{2}+u_{0}-u_{1}\right)\right|_{\Gamma_{s}} .
\end{gathered}
$$

This problem can be considered as a scalar model of the two-body contact problem between a linear elastic unbounded medium and a deformable body allowing some nonlinear monotone stress strain relationship (refer to $[4,12])$. Some similar problems have been considered in $[5,8,9,14]$. The paper [2] introduced a coupled FEMBEM variational inequality of the problem (1)-(5), and considered the corresponding approximation problem. Moreover, an asymptotic error estimate has been derived in that paper.

However, there is no literature to study the numerical method for solving this kind of coupled FEM-BEM variational inequality. In the present paper we introduce an iterative method for solving (1)-(5). This method can be viewed as a variant of the D-N alternative method, which has been applied to solving the Poisson equations in bounded domain and unbounded domain (see $[7,19,20]$ ). It will be shown that the solution sequence generated by this iteration converges to the solution of the coupled system given in [2], and the convergence speed is independent of the mesh size. The new method has obvious advantages: (1) the finite element problem and boundary element problem are solved respectively; (2) the memory requirement was decreased.

This paper is organized as follows. In Section 2, we describe the coupled variational problem in suitable way. In Section 3, a basic iteration method is described, and its convergence results are given. In Section 4, we analyze the convergence rate. In Section 5, we discuss a combination between this iterative method and the steepest descent method.

\section{A COUpled System DERIVEd By FEM AND BEM}

In this section, we describe the variational problems considered by [2]. For convenience, we would like to use the equivalent forms to $(24)$ and $(28)$ in $[2]$.

Let $H^{1}(\Omega), H^{\frac{1}{2}}(\Gamma), H_{0}^{1}(\Omega)$ and $H^{-\frac{1}{2}}(\Gamma)$ denote the usual Sobolev spaces (refer to [10]). We define the (nonlinear) functional $A: H^{1}(\Omega) \times H^{1}(\Omega) \rightarrow \mathbf{R}$ and the linear functional $F: H^{1}(\Omega) \times H^{\frac{1}{2}}(\Gamma) \rightarrow \mathbf{R}$ by

$$
A(u, v)=\int_{\Omega} p(|\nabla u|)(\nabla u)^{t} \cdot \nabla v \mathrm{~d} x, \quad u, v \in H^{1}(\Omega)
$$

and

$$
F(u, \varphi)=\int_{\Omega} f \cdot u \mathrm{~d} x+\int_{\Gamma} t_{0} \cdot \varphi \mathrm{d} s, \quad u \in H^{1}(\Omega), \varphi \in H^{\frac{1}{2}}(\Gamma) .
$$

To define a symmetric and positive definite boundary operator, let $\gamma(x, y)$ be the fundamental solution for the Laplacian, i.e.

$$
\gamma(x, y)=-\frac{1}{2 \pi} \log |x-y|, \quad x, y \in \mathbf{R}^{2}
$$


Set

$$
\begin{gathered}
V \phi(z)=2 \int_{\Gamma} \phi(x) \cdot \gamma(z, x) \mathrm{d} s_{x} \quad\left(V: H^{-\frac{1}{2}}(\Gamma) \rightarrow H^{\frac{1}{2}}(\Gamma)\right), \\
K \phi(z)=2 \int_{\Gamma} \phi(x) \cdot \frac{\partial}{\partial n_{x}} \gamma(z, x) \mathrm{d} s_{x} \quad\left(K: H^{\frac{1}{2}}(\Gamma) \rightarrow H^{\frac{1}{2}}(\Gamma)\right), \\
K^{\prime} \phi(z)=2 \int_{\Gamma} \phi(x) \cdot \frac{\partial}{\partial n_{z}} \gamma(z, x) \mathrm{d} s_{x} \quad\left(K^{\prime}: H^{-\frac{1}{2}}(\Gamma) \rightarrow H^{-\frac{1}{2}}(\Gamma)\right), \\
W \phi(z)=\frac{\partial}{\partial n_{z}} K \phi(z) \quad\left(W: H^{\frac{1}{2}}(\Gamma) \rightarrow H^{-\frac{1}{2}}(\Gamma)\right) .
\end{gathered}
$$

It is well known that $V: H^{-\frac{1}{2}}(\Gamma) \rightarrow H^{\frac{1}{2}}(\Gamma)$ is symmetric and positive definite, and $W: H^{\frac{1}{2}}(\Gamma) \rightarrow H^{-\frac{1}{2}}(\Gamma)$ is symmetric and positive semi-definite under suitable assumption (see $[2,15])$. Thus, the operator $S: H^{\frac{1}{2}}(\Gamma) \rightarrow H^{-\frac{1}{2}}(\Gamma)$ defined by

$$
S=\frac{1}{2}\left(W+\left(K^{\prime}-I\right) V^{-1}(K-I)\right)
$$

is also symmetric and positive definite. Note that the operator $S$ is just the Dirichlet - Neumann map or Steklov-Poincare operator (refer to $[2,18]$ ).

Let $\langle\cdot, \cdot\rangle$ be the $L^{2}$ inner product on $\Gamma$. Set

$$
L(u, \varphi)=F(u, \varphi)+\langle S a, \varphi\rangle, \quad u \in H^{1}(\Omega), \varphi \in H^{\frac{1}{2}}(\Gamma) .
$$

For the function $p(t)$, we make the natural assumption: $p_{1} \leq p(t) \leq p_{2}$ and $\alpha \leq p(t)+t p^{\prime}(t) \leq \beta$ for constants $p_{1}, p_{2}, \alpha, \beta>0$ (refer to [2,11]). Below we fix the constant $a$ in some way to force uniqueness (see [2]).

Let $E=H^{1}(\Omega) \times H^{\frac{1}{2}}(\Gamma)$. Set

$$
D=\left\{(u, \varphi) \in E: u=\varphi+u_{0} \text { on } \Gamma_{t} \text { and } u \leq \varphi+u_{0} \text { a.e. on } \Gamma_{s}\right\}
$$

The variational form of the problem $(1)-(5)$ is: to find $(u, \varphi) \in D$ such that

$$
\begin{gathered}
A(u, v-u)+\langle S \varphi, \varphi-\psi\rangle \geq L(v-u, \varphi-\psi), \\
\forall(v, \psi) \in D .
\end{gathered}
$$

If $(u, \varphi)$ is the solution of (2.1), then we can obtain the solution of (1)-(5) by $u_{1}=u$ and

$$
u_{2}(z)=\frac{1}{2}(K \varphi+V S(\varphi-a))(z)+a, \quad z \in \Omega_{c}
$$

Without loss of generality, we assume that the domain $\Omega$ is a polygon. Let $\Omega$ be divided into some regular quasi-uniform triangles with diameter $h$. Then let $H_{h}^{1}$ denote the finite-dimensional space of continuous and piecewise linear function relating to this partition. The mesh in $\Omega$ leads to a mesh of the boundary, so that we set

Moreover, we may consider $H_{h}^{-\frac{1}{2}}$ as the piecewise constant trial functions. For simplicity of exposition, we assume that $u_{0} \in H_{h}^{\frac{1}{2}}$. Let $E_{h}=H_{h}^{1} \times H_{h}^{\frac{1}{2}}$, and set

$$
D_{h}=\left\{\left(u_{h}, \varphi_{h}\right) \in E_{h}: u_{h}=\varphi_{h}+u_{0} \text { on } \Gamma_{t} \text { and } u_{h} \leq \varphi_{h}+u_{0} \text { a.e. on } \Gamma_{s}\right\} \text {. }
$$

Let

$$
i_{h}: H_{h}^{\frac{1}{2}} \hookrightarrow H^{\frac{1}{2}}(\Gamma)
$$


and

denote the canonical imbedding with its dual $i_{h}^{*}$ and $j_{h}^{*}$

$$
j_{h}: H_{h}^{-\frac{1}{2}} \hookrightarrow H^{-\frac{1}{2}}(\Gamma)
$$

$$
i_{h}^{*}: H^{-\frac{1}{2}}(\Gamma) \hookrightarrow\left(H_{h}^{\frac{1}{2}}\right)^{*}
$$

and

Set

$$
j_{h}^{*}: H^{\frac{1}{2}}(\Gamma) \hookrightarrow\left(H_{h}^{-\frac{1}{2}}\right)^{*} .
$$

$$
S_{h}=\frac{1}{2}\left(i_{h}^{*} W i_{h}+i_{h}^{*}\left(K^{\prime}-I\right) j_{h}\left(j_{h}^{*} V j_{h}\right)^{-1} j_{h}^{*}(K-I) i_{h}\right) .
$$

The discrete problem associated with $(2.1)$ is: to find $\left(u_{h}, \varphi_{h}\right) \in D_{h}$ such that

where

$$
\begin{gathered}
A\left(u_{h}, v_{h}-u_{h}\right)+\left\langle S_{h} \varphi_{h}, \psi_{h}-\varphi_{h}\right\rangle \geq L_{h}\left(v_{h}-u_{h}, \psi_{h}-\varphi_{h}\right), \\
\forall\left(v_{h}, \psi_{h}\right) \in D_{h}
\end{gathered}
$$

$$
L_{h}(v, \psi)=F(v, \psi)+\left\langle S_{h} a, \psi\right\rangle .
$$

It has been shown in [2] that the discrete problem (2.2) (and (2.1)) has a unique solution (by transforming it to an equivalent form). Moreover, an asymptotic estimate of the errors $u_{h}-u$ and $\varphi_{h}-\varphi$ was also derived. If $\left(u_{h}, \varphi_{h}\right)$ is the solution of (2.2), then we can obtain the approximate solution of (1)-(5) by $u_{1 h}=u_{h}$ and

$$
u_{2 h}(z)=\frac{1}{2}\left(K \varphi_{h}+V S\left(\varphi_{h}-a\right)\right)(z)+a, \quad z \in \Omega_{c} .
$$

Remark 2.1. Since the stiffness matrix of the boundary integral operator $S_{h}$ is dense, it is very expensive to solve the variational inequality $(2.2)$ by the existing methods (refer to $[6,19]$ ). For this reason, we will introduce an iterative method to solve (2.2) in the next section.

\section{A BASiC iterative Method}

At first, we describe the algorithm.

For a given $\lambda \in H^{\frac{1}{2}}(\Gamma)$, we define

$$
\hat{H}_{\lambda}^{1}=\left\{v \in H_{h}^{1}: v=\lambda+u_{0} \text { on } \Gamma_{t} \text { and } v \leq \lambda+u_{0} \text { a.e. on } \Gamma_{s}\right\} \text {. }
$$

For a given $w \in H_{h}^{\frac{1}{2}}$, let $\bar{w} \in H_{h}^{1}$ denote the zero extension of $w$ (which equals $w$ on $\Gamma$, and equals zero at the internal nodes of $\Omega$ ).

Algorithm 3.1 Let $\varphi_{h}^{0} \in H_{h}^{\frac{1}{2}}$ be given. When $\varphi_{h}^{n} \in H_{h}^{\frac{1}{2}}$ have been gotten, $\varphi_{h}^{n+1} \in H_{h}^{\frac{1}{2}}$ will be obtained by step $1^{\circ}$ : to find $u_{h}^{n+1} \in \hat{H}_{\varphi_{h}^{n}}^{1}$ such that

$$
A\left(u_{h}^{n+1}, v_{h}-u_{h}^{n+1}\right) \geq\left(f, v_{h}-u_{h}^{n+1}\right), \forall v_{h} \in \hat{H}_{\varphi_{h}^{n}}^{1}
$$

step $2^{\circ}$ : to find $p_{h}^{n+1} \in H_{h}^{\frac{1}{2}}$ such that

$$
\left\langle S_{h} p_{h}^{n+1}, \psi_{h}\right\rangle=\left(f, \overline{\psi_{h}}\right)-A\left(u_{h}^{n+1}, \overline{\psi_{h}}\right)+\left\langle t_{0}+S a, \psi_{h}\right\rangle, \forall \psi_{h} \in H_{h}^{\frac{1}{2}}
$$

step $3^{\circ}$ : set

$$
\varphi_{h}^{n+1}=\theta p_{h}^{n+1}+(1-\theta) \varphi_{h}^{n}, \quad 0<\theta<1 .
$$


Remark 3.1. In essence, the step $1^{\circ}$ and the step $2^{\circ}$ can be regarded respectively as solving a Dirichlet (inequality) problem on $\Omega$ (by FEM) and as solving a Neumann problem on $\Omega_{c}$ (by BEM). In the implementation of the step $2^{\circ}$, it is unnecessary to consider the operator $i_{h}^{*}$ or $j_{h}^{*}$. Instead, only the Galerkin method is used (refer to [1]).

Remark 3.2. The main merits of this algorithm are: (1) the finite element problem and the boundary element problem are solved independently, the variational inequality (3.1) and the boundary integral equation (3.2) can be solved by the existing methods (see $[3,6]) ;(2)$ it has smaller memory requirement than the algorithms to solve globally the variational inequality $(2.2)$.

Now, we give the convergence result of this iteration.

Since $S_{h}: H_{h}^{\frac{1}{2}} \rightarrow\left(H_{h}^{\frac{1}{2}}\right)^{*}$ is a symmetric and positive definite operator, we can define the norm $\|\cdot\|_{S_{h}}=\left(\left\langle S_{h}, \cdot\right\rangle\right)^{\frac{1}{2}}$. Let $\varphi_{h} \in H^{\frac{1}{2}}$ be determined by the variational inequality $(2.2)$, and set $e_{h}^{n}=\varphi_{h}-\varphi_{h}^{n}$.

Theorem 3.1. There exists a constant $c_{0}$ independent of the mesh size $h$, such that when $0<\theta<\frac{1}{c_{0}+1}$, the $D-N$ alternative algorithm is convergent. Moreover, we have

$$
\left\|e_{h}^{n+1}\right\|_{S_{h}}^{2} \leq\left(1-2 \theta+2\left(c_{0}+1\right) \theta^{2}\right)\left\|e_{h}^{n}\right\|_{S_{h}}^{2} .
$$

In particular, when $\theta=\frac{1}{2\left(c_{0}+1\right)}$, the upper bound of (3.4) is minimal and there holds

$$
\left\|e_{h}^{n+1}\right\|_{S_{h}}^{2} \leq\left(1-\frac{1}{2\left(c_{0}+1\right)}\right)\left\|e_{h}^{n}\right\|_{S_{h}}^{2}
$$

Remark 3.3. Set $u_{1 h}^{n}=u_{h}^{n}$ and

$$
u_{2 h}^{n}(z)=\frac{1}{2}\left(K \varphi_{h}^{n}+V S\left(\varphi_{h}^{n}-a\right)\right)(z)+a, \quad z \in \Omega_{c} .
$$

Theorem 3.1 proves that $u_{1 h}^{n}$ and $u_{2 h}^{n}$ converge to $u_{1 h}$ and $u_{2 h}$ respectively. In fact, we have

$$
\left\|u_{1 h}^{n}-u_{1 h}\right\|_{1, \Omega} \leq C\left(1-\frac{1}{2\left(c_{0}+1\right)}\right)^{\frac{n}{2}}\left\|e_{h}^{0}\right\|_{S_{h}},
$$

with $C$ being a constant independent of $h$ and $n$. The error $u_{2 h}^{n}-u_{2 h}$ has a similar estimate, but it involves a particular norm on the unbounded domain $\Omega_{c}$.

\section{Analysis of the CONVERGEnCE RATE}

Since the nonlinear variational inequality is involved, Theorem 3.1 can not be derived by the standard eigenvalue method, which has been used to analyze the convergence rate of the usual iterative method (compare $[7,19])$. The proof of Theorem 3.1 is based on four lemmas.

Lemma 4.1. The variational inequality (2.2) can be decomposed into the following sub-problems

$$
A\left(u_{h}, v_{h}-u_{h}\right) \geq\left(f, v_{h}-u_{h}\right), \quad \forall v_{h} \in \hat{H}_{\varphi_{h}}^{1}
$$

and

$$
\left\langle S_{h} \varphi_{h}, \psi_{h}\right\rangle=\left(f, \bar{\psi}_{h}\right)-A\left(u_{h}, \bar{\psi}_{h}\right)+\left\langle t_{0}+S a, \psi_{h}\right\rangle, \forall \psi_{h} \in H_{h}^{\frac{1}{2}}
$$


Proof. The variational inequality (4.1) follows by setting $\psi_{h}=\varphi_{h}$ in (2.2).

For any $w_{h} \in H_{h}^{\frac{1}{2}}$, we set $v_{h}=u_{h}+\overline{w_{h}}$ (or $u_{h}-\overline{w_{h}}$ ) and $\psi_{h}=\varphi_{h}+w_{h}\left(\right.$ or $\left.\varphi_{h}-w_{h}\right)$ in (2.2). Then, the function $\varphi_{h} \in H_{h}^{\frac{1}{2}}$ satisfies

and

$$
A\left(u_{h}, \overline{w_{h}}\right)+\left\langle S_{h} \varphi_{h}, w_{h}\right\rangle \geq\left(f, \overline{w_{h}}\right)+\left\langle t_{0}+S a, w_{h}\right\rangle
$$

$$
A\left(u_{h},-\overline{w_{h}}\right)+\left\langle S_{h} \varphi_{h},-w_{h}\right\rangle \geq\left(f,-\overline{w_{h}}\right)+\left\langle t_{0}+S a,-w_{h}\right\rangle .
$$

These two inequalities imply the equation (4.2).

Set

$$
\mu_{h}^{n}=p_{h}^{n+1}-\varphi_{h}
$$

Lemma 4.2. The following inequality is valid:

$$
A\left(u_{h}, u_{h}-u_{h}^{n+1}\right)-A\left(u_{h}^{n+1}, u_{h}-u_{h}^{n+1}\right) \leq\left\langle S_{h} \mu_{h}^{n}, e_{h}^{n}\right\rangle .
$$

Proof. It can be verified directly that

$$
\begin{aligned}
\left\langle S_{h} \mu_{h}^{n}, e_{h}^{n}\right\rangle= & \left\langle S_{h} \mu_{h}^{n}, u_{h}-u_{h}^{n+1}\right\rangle+\left\langle S_{h} \mu_{h}^{n}, \varphi_{h}+u_{0}-u_{h}\right\rangle+\left\langle S_{h} \mu_{h}^{n}, u_{h}^{n+1}-\left(\varphi_{h}^{n}+u_{0}\right)\right\rangle \\
= & \left\langle S_{h} \mu_{h}^{n}, u_{h}-u_{h}^{n+1}\right\rangle+\left\langle S_{h} p_{h}^{n+1}, \varphi_{h}+u_{0}-u_{h}\right\rangle-\left\langle S_{h} \varphi_{h}, \varphi_{h}+u_{0}-u_{h}\right\rangle \\
& +\left\langle S_{h} p_{h}^{n+1}, u_{h}^{n+1}-\left(\varphi_{h}^{n}+u_{0}\right)\right\rangle-\left\langle S_{h} \varphi_{h}, u_{h}^{n+1}-\left(\varphi_{h}^{n}+u_{0}\right)\right\rangle .
\end{aligned}
$$

Set $w_{h}=\varphi_{h}+u_{0}-\left.u_{h}\right|_{\Gamma}$. From the definition of $p_{h}^{n+1}$ (see step $2^{\circ}$ ), we have

$$
\begin{aligned}
\left\langle S_{h} p_{h}^{n+1}, \varphi_{h}+u_{0}-u_{h}\right\rangle=\left\langle S_{h} p_{h}^{n+1}, w_{h}\right\rangle= & \left(f, \overline{w_{h}}\right)-A\left(u_{h}^{n+1}, \overline{w_{h}}\right)+\left\langle t_{0}+S a, \overline{w_{h}}\right\rangle \\
= & \left\langle t_{0}+S a, w_{h}\right\rangle-\left[\left(f,-\overline{w_{h}}\right)-A\left(u_{h}^{n+1},-\overline{w_{h}}\right)\right] \\
= & \left\langle t_{0}+S a, w_{h}\right\rangle-\left[\left(f,\left(u_{h}^{n+1}-\overline{w_{h}}\right)-u_{h}^{n+1}\right)\right. \\
& \left.-A\left(u_{h}^{n+1},\left(u_{h}^{n+1}-\overline{w_{h}}\right)-u_{h}^{n+1}\right)\right] .
\end{aligned}
$$

Since $u_{h} \in \hat{H}_{\varphi_{h}}^{1}$, we know that

Note that $u_{h}^{n+1} \in \hat{H}_{\varphi_{h}^{n}}^{1}$, we have

$$
\left.\left.w_{h}\right|_{\Gamma_{t}}=0 \text { and }\left.w_{h}\right|_{\Gamma_{s}} \geq 0 \text { (a.e. on } \Gamma_{s}\right) .
$$

$$
\left.u_{h}^{n+1}\right|_{\Gamma_{t}}=\varphi_{h}^{n}+u_{0} \text { and }\left.u_{h}^{n+1}\right|_{\Gamma_{t}} \leq \varphi_{h}^{n}+u_{0}\left(\text { a.e. on } \Gamma_{s}\right),
$$

by (4.6), this leads to

$$
\left.\left.\left(u_{h}^{n+1}-\overline{w_{h}}\right)\right|_{\Gamma_{t}}=\varphi_{h}^{n}+u_{0} \text { and }\left.\left(u_{h}^{n+1}-\overline{w_{h}}\right)\right|_{\Gamma_{t}} \leq \varphi_{h}^{n}+u_{0} \text { (a.e. on } \Gamma_{s}\right) .
$$

Namely, $u_{h}^{n+1}-\overline{w_{h}} \in \hat{H}_{\varphi_{h}^{n}}^{1}$. Thus, by (4.5) and the definition of $u_{h}^{n+1}$ (see step $1^{\circ}$ ) we obtain

$$
\left\langle S_{h} p_{h}^{n+1}, \varphi_{h}+u_{0}-u_{h}\right\rangle \geq\left\langle t_{0}+S a, w_{h}\right\rangle=\left\langle t_{0}+S a, \varphi_{h}+u_{0}-u_{h}\right\rangle .
$$

Similarly, we can prove

$$
\begin{aligned}
-\left\langle S_{h} \varphi_{h}, u_{h}^{n+1}-\left(\varphi_{h}^{n}+u_{0}\right)\right\rangle & =\left\langle S_{h} \varphi_{h}, \varphi_{h}^{n}+u_{0}-u_{h}^{n+1}\right\rangle \\
& \geq\left\langle t_{0}+S a, \varphi_{h}^{n}+u_{0}-u_{h}^{n+1}\right\rangle .
\end{aligned}
$$

On the other hand, let $\hat{v_{h}} \in H_{h}^{1}$ denote a suitable extension of $\varphi_{h}+u_{0}$ such that $\hat{v_{h}}-u_{h}$ is just the zero extension of $\varphi_{h}+u_{0}-\left.u_{h}\right|_{\Gamma}$. From (4.2), we have

$$
\left.\left\langle S_{h} \varphi_{h}, \varphi_{h}+u_{0}-u_{h}\right\rangle=\left(f, \hat{v_{h}}-u_{h}\right)-A\left(u_{h}, \hat{v_{h}}-u_{h}\right)+\left\langle t_{0}+S a, \varphi_{h}+u_{0}-u_{h}\right)\right\rangle .
$$


It is clear that $\hat{v_{h}} \in \hat{H}_{\varphi_{h}}^{1}$. Thus, by (4.1) and (4.9) we obtain

$$
\left\langle S_{h} \varphi_{h}, \varphi_{h}+u_{0}-u_{h}\right\rangle \leq\left\langle t_{0}+S a, \varphi_{h}+u_{0}-u_{h}\right\rangle
$$

Namely,

$$
-\left\langle S_{h} \varphi_{h}, \varphi_{h}+u_{0}-u_{h}\right\rangle \geq-\left\langle t_{0}+S a, \varphi_{h}+u_{0}-u_{h}\right\rangle .
$$

In an analogous way, we can prove

$$
\begin{aligned}
\left\langle S_{h} p_{h}^{n+1}, u_{h}^{n+1}-\left(\varphi_{h}^{n}+u_{0}\right)\right\rangle= & -\left\langle S_{h} p_{h}^{n+1}, \varphi_{h}^{n}+u_{0}-u_{h}^{n+1}\right\rangle \\
\geq & -\left\langle t_{0}+S a, \varphi_{h}^{n}+u_{0}-u_{h}^{n+1}\right\rangle .
\end{aligned}
$$

Using (4.4), together with (4.7)-(4.8) and (4.10)-(4.11), yields

$$
\left\langle S_{h} \mu_{h}^{n}, e_{h}^{n}\right\rangle \geq\left\langle S_{h} \mu_{h}^{n}, u_{h}-u_{h}^{n+1}\right\rangle=\left\langle S_{h} p_{h}^{n+1}, u_{h}-u_{h}^{n+1}\right\rangle-\left\langle S_{h} \varphi_{h}, u_{h}-u_{h}^{n+1}\right\rangle .
$$

Subtracting (4.2) from (3.2), and choosing $\psi_{h}=\left.\left(u_{h}-u_{h}^{n+1}\right)\right|_{\Gamma}$, we obtain

$$
\left\langle S_{h} p_{h}^{n+1}, u_{h}-u_{h}^{n+1}\right\rangle-\left\langle S_{h} \varphi_{h}, u_{h}-u_{h}^{n+1}\right\rangle=A\left(u_{h}, \overline{e_{h \Gamma}}\right)-A\left(u_{h}^{n+1}, \overline{e_{h \Gamma}}\right)
$$

with $e_{h \Gamma}=\left.\left(u_{h}-u_{h}^{n+1}\right)\right|_{\Gamma}$. It follows by (3.1) and (4.1) that

$$
A\left(u_{h}^{n+1}, w_{h}\right)=\left(f, w_{h}\right), \quad \forall w_{h} \in H_{h}^{1} \cap H_{0}^{1}(\Omega)
$$

and

Hence,

$$
A\left(u_{h}, w_{h}\right)=\left(f, w_{h}\right), \quad \forall w_{h} \in H_{h}^{1} \cap H_{0}^{1}(\Omega) .
$$

Namely,

$$
A\left(u_{h}^{n+1}, \overline{e_{h \Gamma}}-\left(u_{h}-u_{h}^{n+1}\right)\right)=\left(f, \overline{e_{h \Gamma}}-\left(u_{h}-u_{h}^{n+1}\right)\right)=A\left(u_{h}, \overline{e_{h \Gamma}}-\left(u_{h}-u_{h}^{n+1}\right)\right) .
$$

$$
A\left(u_{h}, \overline{e_{h \Gamma}}\right)-A\left(u_{h}^{n+1}, \overline{e_{h \Gamma}}\right)=A\left(u_{h}, u_{h}-u_{h}^{n+1}\right)-A\left(u_{h}^{n+1}, u_{h}-u_{h}^{n+1}\right) .
$$

This, together with (4.12) and (4.13), gives the desired result.

By (4.3) and the monotonicity of the (nonlinear) functional $A(\cdot, \cdot)$ (see [2]), we obtain

Corollary 4.1. We have the inequality

$$
\left\langle S_{h} \mu_{h}^{n}, e_{h}^{n}\right\rangle \geq 0 .
$$

For a given $\lambda \in H_{h}^{\frac{1}{2}}$, let $\widetilde{\lambda} \in H_{h}^{1}$ denote the discrete harmonic extension of $\lambda$.

Lemma 4.3. There exists a positive constant c such that

$$
\left|\widetilde{\mu_{h}^{n}}\right|_{1, \Omega}^{2} \leq c\left\langle S_{h} \mu_{h}^{n}, \mu_{h}^{n}\right\rangle
$$

Proof. Let $H\left(\mu_{h}^{n}\right) \in H^{1}(\Omega)$ denote the (continuous) harmonic extension. Since $\widetilde{\mu_{h}^{n}}$ is just the orthogonal projection of $H\left(\mu_{h}^{n}\right)$, we have

$$
\left|\widetilde{\mu_{h}^{n}}\right|_{1, \Omega}^{2} \leq\left|H\left(\mu_{h}^{n}\right)\right|_{1, \Omega}^{2}
$$

It follows by the well-known property of $H\left(\mu_{h}^{n}\right)$ that there is a positive constant $c_{1}$ independent of $h$, such that

$$
\left|H\left(\mu_{h}^{n}\right)\right|_{1, \Omega}^{2} \leq c_{1}\left\|\mu_{h}^{n}\right\|_{\frac{1}{2}, \Gamma}^{2} .
$$

Thus, by (4.17) and Lemma 5.1 in [2], we obtain

$$
\left|\widetilde{\mu_{h}^{n}}\right|_{1, \Omega}^{2} \leq c_{1}\left\|\mu_{h}^{n}\right\|_{\frac{1}{2}, \Gamma}^{2} \leq c\left\langle S_{h} \mu_{h}^{n}, \mu_{h}^{n}\right\rangle .
$$


Lemma 4.4. There exists a positive constant $c_{0}$ such that

$$
\left\langle S_{h} \mu_{h}^{n}, \mu_{h}^{n}\right\rangle \leq c_{0}\left\langle S_{h} e_{h}^{n}, e_{h}^{n}\right\rangle
$$

Proof. Subtracting (4.2) from (3.2), and choosing $\psi_{h}=\mu_{h}^{n}$, yields

$$
\begin{aligned}
\left\langle S_{h} \mu_{h}^{n}, \mu_{h}^{n}\right\rangle & =\left\langle S_{h} p_{h}^{n+1}, \mu_{h}^{n}\right\rangle-\left\langle S_{h} \varphi_{h}, \mu_{h}^{n}\right\rangle \\
& =A\left(u_{h}, \overline{\mu_{h}^{n}}\right)-A\left(u_{h}^{n+1}, \overline{\mu_{h}^{n}}\right) .
\end{aligned}
$$

Since $\left.\left(\overline{\mu_{h}^{n}}-\widetilde{\mu_{h}^{n}}\right)\right|_{\Gamma}=0$, we have (refer to $(4.14)$ )

$$
A\left(u_{h}, \overline{\mu_{h}^{n}}\right)-A\left(u_{h}^{n+1}, \overline{\mu_{h}^{n}}\right)=A\left(u_{h}, \widetilde{\mu_{h}^{n}}\right)-A\left(u_{h}^{n+1}, \widetilde{\mu_{h}^{n}}\right) .
$$

Substituting the above equality into (4.19), yields

$$
\left\langle S_{h} \mu_{h}^{n}, \mu_{h}^{n}\right\rangle=A\left(u_{h}, \widetilde{\mu_{h}^{n}}\right)-A\left(u_{h}^{n+1}, \widetilde{\mu_{h}^{n}}\right) .
$$

On the other hand, by the assumptions to the function $p(t)$ (see Sect. 2), we can prove (refer to $[2,11]$ )

$$
A\left(u_{h}, \widetilde{\mu_{h}^{n}}\right)-A\left(u_{h}^{n+1}, \widetilde{\mu_{h}^{n}}\right) \leq \beta\left|u_{h}-u_{h}^{n+1}\right|_{1, \Omega} \cdot\left|\widetilde{\mu_{h}^{n}}\right|_{1, \Omega}
$$

and

Hence, by (4.20)-(4.22), we obtain

$$
\alpha\left|u_{h}-u_{h}^{n+1}\right|_{1, \Omega}^{2} \leq A\left(u_{h}, u_{h}-u_{h}^{n+1}\right)-A\left(u_{h}^{n+1}, u_{h}-u_{h}^{n+1}\right) .
$$

$$
\left\langle S_{h} \mu_{h}^{n}, \mu_{h}^{n}\right\rangle \leq \alpha^{-\frac{1}{2}} \beta\left[A\left(u_{h}, u_{h}-u_{h}^{n+1}\right)-A\left(u_{h}^{n+1}, u_{h}-u_{h}^{n+1}\right)\right]^{\frac{1}{2}} \cdot\left|\widetilde{\mu_{h}^{n}}\right|_{1, \Omega}
$$

Furthermore, it follows by (4.3) that

$$
\left\langle S_{h} \mu_{h}^{n}, \mu_{h}^{n}\right\rangle \leq \alpha^{-\frac{1}{2}} \beta\left\langle S_{h} \mu_{h}^{n}, e_{h}^{n}\right\rangle^{\frac{1}{2}} \cdot\left|\widetilde{\mu_{h}^{n}}\right|_{1, \Omega}
$$

which, together with (4.16), yields

$$
\left\langle S_{h} \mu_{h}^{n}, \mu_{h}^{n}\right\rangle \leq \alpha^{-1} \beta^{2} c\left\langle S_{h} \mu_{h}^{n}, e_{h}^{n}\right\rangle .
$$

Since $S_{h}: H_{h}^{\frac{1}{2}} \rightarrow\left(H_{h}^{\frac{1}{2}}\right)^{*}$ is a symmetric and positive definite, by the Cauchy-Schwarz inequality, we obtain

$$
\left\langle S_{h} \mu_{h}^{n}, \mu_{h}^{n}\right\rangle \leq \alpha^{-1} \beta^{2} c\left\langle S_{h} \mu_{h}^{n}, \mu_{h}^{n}\right\rangle^{\frac{1}{2}} \cdot\left\langle S_{h} e_{h}^{n}, e_{h}^{n}\right\rangle^{\frac{1}{2}}
$$

Namely,

$$
\left\langle S_{h} \mu_{h}^{n}, \mu_{h}^{n}\right\rangle \leq \alpha^{-2} \beta^{4} c^{2}\left\langle S_{h} e_{h}^{n}, e_{h}^{n}\right\rangle .
$$

This means that the inequality (4.18) is valid with $c_{0}=\alpha^{-2} \beta^{2} c^{2}$.

Now, we can prove our main result easily.

Proof of Theorem 3.1. From (3.3), we have

$$
e_{h}^{n+1}=e_{h}^{n}-\theta g_{h}^{n}
$$

where $g_{h}^{n}=p_{h}^{n+1}-\varphi_{h}^{n}$. It follows by (4.24) that

$$
\left\|e_{h}^{n+1}\right\|_{S_{h}}^{2}=\left\|e_{h}^{n}\right\|_{S_{h}}^{2}-2 \theta\left\langle S_{h} e_{h}^{n}, g_{h}^{n}\right\rangle+\theta^{2}\left\|g_{h}^{n}\right\|_{S_{h}}^{2} .
$$


Namely,

$$
\left\|e_{h}^{n+1}\right\|_{S_{h}}^{2}=\left(1-2 \theta \frac{\left\langle S_{h} e_{h}^{n}, g_{h}^{n}\right\rangle}{\left\|e_{h}^{n}\right\|_{S_{h}}^{2}}+\theta^{2} \frac{\left\|g_{h}^{n}\right\|_{S_{h}}^{2}}{\left\|e_{h}^{n}\right\|_{S_{h}}^{2}}\right)\left\|e_{h}^{n}\right\|_{S_{h}}^{2} \quad\left(e_{h}^{n} \neq 0\right) .
$$

By Corollary 4.1, yields (note that $g_{h}^{n}=e_{h}^{n}+\mu_{h}^{n}$ )

$$
\left\langle S_{h} e_{h}^{n}, g_{h}^{n}\right\rangle=\left\|e_{h}^{n}\right\|_{S_{h}}^{2}+\left\langle S_{h} e_{h}^{n}, \mu_{h}^{n}\right\rangle \geq\left\|e_{h}^{n}\right\|_{S_{h}}^{2}
$$

Moreover, it follows by Lemma 4.4 that

$$
\begin{aligned}
\left\|g_{h}^{n}\right\|_{S_{h}}^{2} & =\left\|\mu_{h}^{n}+e_{h}^{n}\right\|_{S_{h}}^{2} \\
& \leq 2\left(\left\|\mu_{h}^{n}\right\|_{S_{h}}^{2}+\left\|e_{h}^{n}\right\|_{S_{h}}^{2}\right) \\
& \left.\leq 2\left(c_{0}+1\right)\left\|e_{h}^{n}\right\|_{S_{h}}^{2}\right) .
\end{aligned}
$$

Using (4.26), together with (4.27) and (4.28), we deduce to (3.4).

It is clear that when $0<\theta<\frac{1}{c_{0}+1}$, we have

$$
\left\|e_{h}^{n+1}\right\|_{S_{h}}^{2}<\left\|e_{h}^{n}\right\|_{S_{h}}^{2}
$$

It can be verified directly that when $\theta=\frac{1}{2\left(c_{0}+1\right)}$, the function

$$
g(\theta)=1-2 \theta+2\left(c_{0}+1\right) \theta^{2}
$$

reaches the minimum value $1-\frac{1}{2\left(c_{0}+1\right)}$.

Remark 4.1. Some iterative methods for nonlinear minimization problems have been developed by [16,17]. It is easy to see that the methods as well as the convergence results can be extended to the case of nonlinear equation. On the other hand, the unknown $\varphi_{h}$ satisfies a nonlinear equation, and Algorithm 3.1 can be interpreted as a preconditioned Richardson iteration (refer to the next section). Thus, Theorem 3.1 may be derived by using the extended results.

\section{A combination Between the Algorithm 3.1 And the Steepest Descent method}

In most applications, it is difficult to estimate exactly the constant $c$ in (4.16) (or $c_{0}$ in Theorem 3.1). To solve this problem, we consider the steepest descent method, by which we can determine a (variable) relaxation parameter $\theta_{n}$ in the $n-t h$ iteration (refer to $[6,13]$ ).

For a given $\lambda \in H_{h}^{\frac{1}{2}}$, let $\Psi(\lambda) \in \hat{H}_{\lambda}^{1}$ denote the solution of the variational inequality

$$
A(\Psi(\lambda), v-\Psi(\lambda)) \geq(f, v-\Psi(\lambda)), \forall v \in \hat{H}_{\lambda}^{1} .
$$

We define the (nonlinear) operator $S_{\Omega}: H_{h}^{\frac{1}{2}} \rightarrow\left(H_{h}^{\frac{1}{2}}\right)^{*}$ by

$$
\left\langle S_{\Omega} \lambda, \mu\right\rangle=A(\Psi(\lambda), \bar{\mu})-(f, \bar{\mu}), \forall \mu \in H_{h}^{\frac{1}{2}} .
$$

Since $\Psi\left(\varphi_{h}\right)=u_{h}$, it follows by Lemma 4.1 that $\varphi_{h}$ satisfies the interface equation

$$
\hat{S} \varphi_{h}=g,
$$

where

$$
\hat{S}=S_{\Omega}+S_{h} \text { and } g=t_{0}+S_{h} a .
$$


It is clear that $p_{h}^{n+1}=S_{h}^{-1}\left(g-S_{\Omega} \varphi_{h}^{n}\right)$. Thus, the algorithm 3.1 given in Section 3 can be regarded as the preconditioned Richardson iteration

$$
\varphi_{h}^{n+1}=\varphi_{h}^{n}+\theta S_{h}^{-1}\left(g-\hat{S} \varphi_{h}^{n}\right) .
$$

Now, the (variable) parameter $\theta\left(=\theta_{n}\right)$ in (5.3) is chosen such that the function

$$
G(\theta)=\left\|\hat{S} \varphi_{h}-\hat{S} \varphi_{h}^{n+1}\right\|_{S_{h}^{-1}}^{2}
$$

reaches its minimum value when $\theta=\theta_{n}$.

Some algorithms of determining the parameter $\theta_{n}$ are given in [13]. For the combined method, the main cost still results from the Algorithm 3.1 described in Section 3.

Let $\left\{\varphi_{h}^{n}\right\}$ denote the solution sequence generated by this method.

Theorem 5.1. For the combined method, we have the following convergence result:

$$
\left\|\varphi_{h}-\varphi_{h}^{n}\right\|_{S_{h}}^{2} \leq\left(1-\frac{1}{2\left(c_{0}+1\right)}\right)^{n}\left\|\hat{S} \varphi_{h}-\hat{S} \varphi_{h}^{0}\right\|_{S_{h}^{-1}}^{2}
$$

where $c_{0}$ is the constant given in the last section.

To prove this theorem, we need two lemmas.

The following result can be proved like (4.3) (refer to Cor. 4.1).

Lemma 5.1. The following inequality is valid for any $\lambda, \mu \in H_{h}^{\frac{1}{2}}$

$$
\left\langle\lambda-\mu, S_{\Omega} \lambda-S_{\Omega} \mu\right\rangle \geq A(\Psi(\lambda), \Psi(\lambda)-\Psi(\mu))-A(\Psi(\mu), \Psi(\lambda)-\Psi(\mu)) \geq 0 .
$$

Lemma 5.2. For any $\lambda, \mu \in H_{h}^{\frac{1}{2}}$, we have

$$
\left\|S_{\Omega} \lambda-S_{\Omega} \mu\right\|_{S_{h}^{-1}}^{2} \leq c_{0}\|\lambda-\mu\|_{S_{h}}^{2} .
$$

Proof. From the definition of the operator $S_{\Omega}$, we have

$$
\left\|S_{\Omega} \lambda-S_{\Omega} \mu\right\|_{S_{h}^{-1}}^{2}=A(\Psi(\lambda), \widetilde{w})-A(\Psi(\mu), \widetilde{w}),
$$

where

$$
w=S_{h}^{-1}\left(S_{\Omega} \lambda-S_{\Omega} \mu\right)
$$

Thus, we can prove, in an analogous way with (4.18), that

$$
\left\|S_{\Omega} \lambda-S_{\Omega} \mu\right\|_{S_{h}^{-1}}^{2} \leq \alpha^{-1} \beta^{2} c A(\Psi(\lambda), \Psi(\lambda)-\Psi(\mu))-A(\Psi(\mu), \Psi(\lambda)-\Psi(\mu)) .
$$

On the other hand, it follows by (5.5) that

$$
\begin{aligned}
A(\Psi(\lambda), \Psi(\lambda)-\Psi(\mu))-A(\Psi(\mu), \Psi(\lambda)-\Psi(\mu)) & \leq\left\langle\lambda-\mu, S_{\Omega} \lambda-S_{\Omega} \mu\right\rangle \\
& \leq\left\|S_{h}^{\frac{1}{2}}(\lambda-\mu)\right\| \cdot\left\|S_{h}^{-\frac{1}{2}}\left(S_{\Omega} \lambda-S_{\Omega} \mu\right)\right\| .
\end{aligned}
$$

Using (5.7) and (5.8), we deduce to (5.6).

Proof of Theorem 5.1. It follows, by a well-known identity, that

$$
\left\|\hat{S} \varphi_{h}-\hat{S} \varphi_{h}^{n+1}\right\|_{S_{h}^{-1}}^{2}-\left\|\hat{S} \varphi_{h}-\hat{S} \varphi_{h}^{n}\right\|_{S_{h}^{-1}}^{2}=2\left\langle\hat{S} \varphi_{h}-\hat{S} \varphi_{h}^{n}, \hat{S} \varphi_{h}^{n}-\hat{S} \varphi_{h}^{n+1}\right\rangle_{S_{h}^{-1}}+\left\|\hat{S} \varphi_{h}^{n}-\hat{S} \varphi_{h}^{n+1}\right\|_{S_{h}^{-1}}^{2} .
$$


Since

we have

$$
\varphi_{h}^{n}-\varphi_{h}^{n+1}=-\theta_{n} S_{h}^{-1}\left(\hat{S} \varphi_{h}-\hat{S} \varphi_{h}^{n}\right)
$$

$$
\left\langle\hat{S} \varphi_{h}-\hat{S} \varphi_{h}^{n}, \hat{S} \varphi_{h}^{n}-\hat{S} \varphi_{h}^{n+1}\right\rangle_{S_{h}^{-1}}=-\theta^{-1}\left\langle\varphi_{h}^{n}-\varphi_{h}^{n+1}, \hat{S} \varphi_{h}^{n}-\hat{S} \varphi_{h}^{n+1}\right\rangle .
$$

The inequality (5.5) infers that

$$
\left\langle\varphi_{h}^{n}-\varphi_{h}^{n+1}, S_{\Omega} \varphi_{h}^{n}-S_{\Omega} \varphi_{h}^{n+1}\right\rangle \geq 0
$$

Thus (note that $\hat{S}=S_{\Omega}+S_{h}$ )

$$
\begin{aligned}
\left\langle\varphi_{h}^{n}-\varphi_{h}^{n+1}, \hat{S} \varphi_{h}^{n}-\hat{S} \varphi_{h}^{n+1}\right\rangle & \geq\left\langle\varphi_{h}^{n}-\varphi_{h}^{n+1}, S_{h}\left(\varphi_{h}^{n}-\varphi_{h}^{n+1}\right)\right\rangle \\
& =\left(\theta_{n}\right)^{2}\left\|\hat{S} \varphi_{h}-\hat{S} \varphi_{h}^{n}\right\|_{S_{h}^{-1}}^{2}
\end{aligned}
$$

This, together with (5.10), leads to

$$
\left\langle\hat{S} \varphi_{h}-\hat{S} \varphi_{h}^{n}, \hat{S} \varphi_{h}^{n}-\hat{S} \varphi_{h}^{n+1}\right\rangle_{S_{h}^{-1}} \leq-\theta\left\|\hat{S} \varphi_{h}-\hat{S} \varphi_{h}^{n}\right\|_{S_{h}^{-1}}^{2} .
$$

On the other hand, it follows by (5.6) that (note that $\hat{S}=S_{\Omega}+S_{h}$ )

$$
\begin{aligned}
\left\|\hat{S} \varphi_{h}^{n}-\hat{S} \varphi_{h}^{n+1}\right\|_{S_{h}^{-1}}^{2} & \leq 2\left(\left\|S_{\Omega} \varphi_{h}^{n}-S_{\Omega} \varphi_{h}^{n+1}\right\|_{S_{h}^{-1}}^{2}+\left\|\varphi_{h}^{n}-\varphi_{h}^{n+1}\right\|_{S_{h}}^{2}\right) \\
& \left.\leq 2\left(1+c_{0}\right)\left\|\varphi_{h}^{n}-\varphi_{h}^{n+1}\right\|_{S_{h}}^{2}\right) \\
& =2\left(1+c_{0}\right) \theta_{n}^{2}\left\|\hat{S} \varphi_{h}-\hat{S} \varphi_{h}^{n}\right\|_{S_{h}^{-1}}^{2} .
\end{aligned}
$$

Using (5.9), together with (5.12) and (5.13), yields

$$
\left\|\hat{S} \varphi_{h}-\hat{S} \varphi_{h}^{n+1}\right\|_{S_{h}^{-1}}^{2} \leq\left(1-2 \theta_{n}+2\left(1+c_{0}\right) \theta_{n}^{2}\right)\left\|\hat{S} \varphi_{h}-\hat{S} \varphi_{h}^{n}\right\|_{S_{h}^{-1}}^{2} .
$$

Since the parameter $\theta_{n}$ is chosen such that

$$
\left\|\hat{S} \varphi_{h}-\hat{S} \varphi_{h}^{n+1}\right\|_{S_{h}^{-1}}^{2}
$$

reaches its minimum value, by $(5.14)$ we obtain $\left(\right.$ set $\left.\theta_{n}=\frac{1}{2\left(c_{0}+1\right)}\right)$

$$
\left\|\hat{S} \varphi_{h}-\hat{S} \varphi_{h}^{n+1}\right\|_{S_{h}^{-1}}^{2} \leq\left(1-\frac{1}{2\left(c_{0}+1\right)}\right)\left\|\hat{S} \varphi_{h}-\hat{S} \varphi_{h}^{n}\right\|_{S_{h}^{-1}}^{2}, \quad n=0,1, \cdots
$$

Hence,

$$
\left\|\hat{S} \varphi_{h}-\hat{S} \varphi_{h}^{n}\right\|_{S_{h}^{-1}}^{2} \leq\left(1-\frac{1}{2\left(c_{0}+1\right)}\right)^{n}\left\|\hat{S} \varphi_{h}-\hat{S} \varphi_{h}^{0}\right\|_{S_{h}^{-1}}^{2} .
$$

To prove (5.4), we only need to verify

$$
\left\|\hat{S} \varphi_{h}-\hat{S} \varphi_{h}^{n}\right\|_{S_{h}^{-1}}^{2} \geq\left\|\varphi_{h}-\varphi_{h}^{n}\right\|_{S_{h}}^{2} .
$$

In fact, it is clear that

$$
\begin{aligned}
\left\|\hat{S} \varphi_{h}-\hat{S} \varphi_{h}^{n}\right\|_{S_{h}^{-1}}^{2} & =\left\|\left(S_{\Omega} \varphi_{h}-S_{\Omega} \varphi_{h}^{n}\right)+S_{h}\left(\varphi_{h}-\varphi_{h}^{n}\right)\right\|_{S_{h}^{-1}}^{2} \\
& \geq 2\left\langle S_{\Omega} \varphi_{h}-S_{\Omega} \varphi_{h}^{n}, \varphi_{h}-\varphi_{h}^{n}\right\rangle+\left\|S_{h}\left(\varphi_{h}-\varphi_{h}^{n}\right)\right\|_{S_{h}^{-1}}^{2}
\end{aligned}
$$

which, together with (5.5), gives (5.15). 
Remark 5.1. Since the (nonlinear) operator $S_{\Omega}$ can not be expressed explicitly, it is impossible to calculate directly its Gateaux derivative (or Frechet derivative). Thus, Theorem 5.1 can not be proved in the usual way (compare [13] and [16]).

Remark 5.2. Since the convergence speed of the methods discussed in this paper is independent of the mesh size $h$, it is not sensitive to the errors of the solutions $u_{h}^{n}, p_{h}^{n}$ (and the parameter $\theta_{n}$ ). The analysis to this dependence is standard (refer to [16]).

Remark 5.3. A similar algorithm with Algorithm 3.1 has been designed for solving linear elliptic problems in unbounded domains in [19], where the efficiency of such algorithm was confirmed by numerical experiments.

Acknowledgements. The authors wish to thank two anonymous referees for many constructive comments which lead to a great improvement of the results and the presentation of the paper.

\section{REFERENCES}

[1] C. Carstensen, Interface problem in holonomic elastoplasticity. Math. Methods Appl. Sci. 16 (1993) 819-835.

[2] C. Carstensen and J. Gwinner, FEM and BEM coupling for a nonlinear transmission problem with Signorini contact. SIAM J. Numer. Anal. 34 (1997) 1845-1864.

[3] C. Carstensen, M. Kuhn and U. Langer, Fast parallel solvers for symmetric boundary element domain decomposition equations. Numer. Math. 79 (1998) 321-347.

[4] M. Costabel and E. Stephan, Coupling of finite and boundary element methods for an elastoplastic interface problem. SIAM J. Numer. Anal. 27 (1990) 1212-1226.

[5] G. Gatica and G. Hsiao, On the coupled BEM and FEM for a nonlinear exterior Dirichlet problem in $R^{2}$. Numer. Math. 61(1992) 171-214.

[6] R. Glowinski, Numerical methods for nonlinear variational problems. Springer-Verlag, New York (1984).

[7] R. Glowinski, G. Golub, G. Meurant and J. Periaux, Eds., Proc. of the the First international symposium on domain decomposition methods for PDEs. SIAM Philadelphia (1988).

[8] Q. Hu and D. Yu, A solution method for a certain interface problem in unbounded domains. Computing 67 (2001) 119-140.

[9] N. Kikuchi and J. Oden, Contact problem in elasticity: a study of variational inequalities and finite element methods. SIAM, Philadelphia (1988).

[10] J. Lions and E. Magenes, Non-homogeneous boundary value problems and applications, Vol. I. Springer-Verlag (1972).

[11] P. Mund and E. Stephan, An adaptive two-level method for the coupling of nonlinear FEM-BEM equations, SIAM J. Numer. Anal. 36 (1999) 1001-1021.

[12] J. Necas, Introduction to the theory of nonlinear elliptic equations. Teubner, Texte 52, Leipzig (1983).

[13] E. Polak, Computational methods in optimization. Academic Press, New York (1971).

[14] J. Schoberl, Solving the Signorini problem on the basis of domain decomposition techniques. Computing 60 (1998) 323-344.

[15] E. Stephan, W. Wendland and G. Hsiao, On the integral equation method for the plane mixed boundary value problem of the Laplacian. Math. Methods Appl. Sci. 1 (1979) 265-321.

[16] X. Tai and M. Espedal, Rate of convergence of some space decomposition methods for linear and nonlinear problems. SIAM J. Numer. Anal. 35 (1998) 1558-1570.

[17] X. Tai and J. Xu, Global convergence of space correction methods for convex optimization problems. Math. Comp. 71 (2002) $105-122$.

[18] D. Yu, The relation between the Steklov-Poincare operator, the natural integral operator and Green functions. Chinese J. Numer. Math. Appl. 17 (1995) 95-106.

[19] D. Yu, Discretization of non-overlapping domain decomposition method for unbounded domains and its convergence. Chinese J. Numer. Math. Appl. 18 (1996) 93-102.

[20] D. Yu, Natural Boundary Integral Method and Its Applications. Science Press/Kluwer Academic Publishers, Beijing/New York (2002). 